\title{
Phenocopies in melanoma-prone families with germ-line CDKN2A mutations
}

\author{
Hildur Helgadottir, MD, PhD ${ }^{1}$, Håkan Olsson, $M D, \mathrm{PhD}^{2}$, Margaret A. Tucker, $\mathrm{MD}^{3}$, \\ Xiaohong R. Yang, $\mathrm{PhD}^{3}$, Veronica Höiom, $\mathrm{PhD}^{1}$ and Alisa M. Goldstein, $\mathrm{PhD}^{3}$
}

Purpose: Carriers of $C D K N 2 A$ mutations have high risks of melanoma and certain other cancers. In this study we examined the occurrence of tumors among CDKN2A wild type (wt) members of melanoma-prone families with $C D K N 2 A$ mutations.

Methods: Swedish and US melanoma-prone families with $C D K N 2 A$ mutations were included. Data was collected on tumors diagnosed among family members. Among the CDKN2A mutated families, members with $C D K N 2 A$ wt status who were diagnosed with melanoma were designated phenocopies.

Results: Of patients with melanoma in the CDKN2A mutated families $(n=266), 7.1 \%$, were seen among members with $C D K N 2 A$ wt status (phenocopy rate). Among the CDKN2A wt family members of the CDKN2A mutated families $(n=256), 7.4 \%$ were diagnosed with melanoma. The prospective relative risk for melanomas was significantly higher among the CDKN2A wt subjects compared with population-based controls (7.4 (95\% confidence interval 1.7-33.2)), while no elevated risks of nonmelanoma cancers were seen and their offspring did not have significantly elevated risks of melanoma or other cancers.

Conclusion: Members of CDKN2A mutation carrying families who test negative for their family's mutation have moderately increased risk for melanoma and should, in addition to being considered for continuing dermatologic surveillance, be encouraged to follow sun safety recommendations and practice skin self-exams.

Genet Med advance online publication 7 December 2017

Key Words: cancer; CDKN2A gene mutations; familial melanoma; phenocopies; wild type (wt)

\section{INTRODUCTION}

Carriers of pathogenic mutations in the CDKN2A tumor suppressor gene have high risks of melanoma and certain other cancers. ${ }^{1-3}$ Similar to other cancer susceptibility genes like BRCA1 and BRCA2, some cancer patients in families with a mutation do not carry their family's mutation and are designated phenocopies. ${ }^{4-7}$ Phenocopies may result from multiple factors, including risk-modifying genes, environmental effects, ascertainment bias, follow-up, or chance. Until now there has been limited knowledge about phenocopies in $C D K N 2 A$ mutated families. It is thus uncertain what advice noncarriers in CDKN2A mutated melanoma families should be given concerning their risk of melanomas or other cancers. In this study we examined the occurrence of cancers among CDKN2A wild type (wt) members of Swedish and US melanoma-prone families with $C D K N 2 A$ mutations.

\section{MATERIALS AND METHODS}

In Sweden, melanoma-prone families were identified through a national preventive program. ${ }^{2,8}$ Family members were invited to undergo germ-line $C D K N 2 A$ mutation analysis for the study. Informed consent was obtained from all participants and the study was approved by local ethical review boards. The national 10-digit personal identity number of CDKN2A genotyped family members was linked with the Swedish Cancer Registry, the Multi-Generation Registry, and the Population Registry. In the Cancer Registry, established in 1958, all cancers diagnosed in Sweden are registered (except for basal cell carcinomas). ${ }^{9}$ The Multi-Generation Registry contains connections between all individuals born after 1931 and their biological parents. ${ }^{10}$ The registry linkage enabled the identification of comprehensive data on census and cancer diagnoses among the CDKN2A genotyped family members, their offspring, and population-based matched controls (case to control ratio 1:10). Follow-up started on the date when the first family member was identified in each family (same date in family members and corresponding controls). Follow-up ended at the date of death, emigration, or census date of 31 December 2011.

In the United States, melanoma-prone families with CDKN2A mutations were part of a non-population-based family study that has been previously described. ${ }^{1,11}$ Briefly, participating families were referred by health-care professionals or through self-referrals. Written informed consent was obtained prior to participation under a National Cancer Institute institutional review board-approved protocol. All family members willing to participate were clinically evaluated, including complete skin examination and routine

${ }^{1}$ Department of Oncology and Pathology, Karolinska Institutet and Karolinska University Hospital, Stockholm, Sweden; ${ }^{2}$ Department of Oncology, Lund University and Skåne University Hospital, Lund, Sweden; ${ }^{3}$ Division of Cancer Epidemiology and Genetics, National Cancer Institute, National Institutes of Health, Bethesda, Maryland, USA. Correspondence: Hildur Helgadottir (hildur.helgadottir@sll.se)

Submitted 24 August 2017; accepted 30 October 2017; advance online publication 7 December 2017. doi:10.1038/gim.2017.216 
Table 1 Frequencies of phenocopies ${ }^{a}$ in Swedish and US melanoma families with CDKN2A mutations

\begin{tabular}{|c|c|c|c|}
\hline & $\begin{array}{l}\text { Swedish families } \\
n / n(\%)\end{array}$ & $\begin{array}{l}\text { US families } \\
n / n(\%)\end{array}$ & $\begin{array}{l}\text { Total } \\
n / n(\%)\end{array}$ \\
\hline No. families with phenocopies/All CDKN2A mutated families & $7 / 34(20.6 \%)$ & $7 / 27(25.9 \%)$ & $14 / 61(22.9 \%)$ \\
\hline No. phenocopies/All melanoma cases in CDKN2A mutated families & $9 / 116(7.8 \%)$ & $10 / 150(6.7 \%)$ & $19 / 266(7.1 \%)$ \\
\hline No. phenocopies/All CDKN2A wt family members in CDKN2A mutated families & $9 / 128(7.0 \%)$ & $10 / 128(7.8 \%)$ & $19 / 256(7.4 \%)$ \\
\hline
\end{tabular}

${ }^{a}$ CDKN2A wild type (wt) melanoma cases from CDKN2A mutated melanoma families.

medical history. Blood was collected primarily for genetic studies. All families were Caucasian and resided in various regions of the United States. The families have been prospectively followed for up to 40 years starting in the 1970s. Follow-up included periodic clinical evaluation and regular requests for updated medical information including the occurrence of cancer. All cancer diagnoses reported were confirmed by review of histologic materials, local pathology reports, medical records, or death certificates. At both sites, mutation testing was performed in available melanoma patients from each family. If a CDKN2A mutation was detected, all available first-degree relatives of cases were tested.

Using data from the Swedish registries, prospective relative risks for melanomas, squamous cell skin cancers, and nonskin cancers were calculated from incidence rates (number of cancers/person-years). All statistical tests were two-sided. Statistical analyses were performed with StatSoft Statistica software, version 10 (StatSoft, Palo Alto, CA, USA).

\section{RESULTS}

Among CDKN2A mutated families, members with CDKN2A wt status, diagnosed with melanoma, were defined as phenocopies. In Sweden and the United States, 34 and 27 melanoma families were identified with pathogenic CDKN2A mutations, of whom 7 (20.6\%) and 7 (25.9\%) families, respectively, had phenocopies (Table 1). The CDKN2A mutations in the families with phenocopies are shown in Supplementary Table S1 online. In total, 116 and 150 patients with melanoma (invasive and in situ) were documented in the Swedish and US CDKN2A mutated families, respectively, of whom $9(7.8 \%)$ and $10(6.7 \%)$, respectively, were seen among members with $C D K N 2 A$ wt status, representing the phenocopy rates in these families. Among CDKN2A wt family members within the CDKN2A mutated families, melanomas were diagnosed among 7.0\% and $7.8 \%$ of the family members who participated in the study, respectively, at each site.

The median age at diagnosis of melanoma in the phenocopies was 47 and 46 years in the Swedish and US families, respectively (Table 2 ). The phenocopy melanoma patients were seen at similar frequencies among women and men. A greater percentage of phenocopy melanoma cases were diagnosed before their family was identified at each of the sites. The vast majority of phenocopies were diagnosed with only one primary melanoma; only one case from Sweden (5.3\% of all 19 phenocopies at both sites) had multiple primary melanomas. One US phenocopy case died from melanoma while the remaining phenocopy patients at both sites were alive at the end of follow-up. One US phenocopy case was diagnosed with squamous cell skin cancers and two with non-skin cancers (breast and colon cancer). In total, 43 children of phenocopies, with available follow-up data, were identified (median age at end of follow-up was 25, range 3-57 years). Among these children there was one diagnosis of squamous cell skin cancers but no diagnoses of melanomas or non-skin cancers.

Using data from Swedish registries, cancer risks were calculated in CDKN2A wt members $(n=128)$ of Swedish CDKN2A mutated families (Supplementary Table S2). Median age of the CDKN2A wt individuals at baseline was 33 years (range 3-79 years). At baseline seven CDKN2A wt individuals had been diagnosed with melanoma, which was significantly greater than among age and sex matched population-based controls $(P<0.001)$. In contrast, at baseline, there was no significant increase in the frequencies of other cancers among the CDKN2A wt individuals, compared with controls. The median age at end of follow-up was 51 years (range 22-88). During follow-up, three subjects were diagnosed with melanoma among the CDKN2A wt individuals, resulting in a significantly increased prospective risk for melanoma compared with controls (7.4 (95\% confidence interval 1.7-33.2)). Similar to baseline, there were no significant differences in prospective risks for other cancers in the CDKN2A wt individuals compared with controls.

From the Swedish Multi-Generation Registry, 185 children of CDKN2A wt family members were identified, of whom $37.3 \%$ were born during the follow-up period (Supplementary Table S2). Median age of the children at the end of follow-up was 22 (range 1-64). One child of a CDKN2A wt subject was diagnosed with a melanoma at baseline, but no melanomas were diagnosed during the follow-up period. Although there was limited power because of the young ages of the children, there were no statistically significant differences in the frequencies or risks of melanomas or other cancers diagnosed at baseline or in the prospective period. Additionally, at the end of follow-up, among 109 grandchildren of CDKN2A wt family members, no melanomas or other cancers had been diagnosed (median age 15 years, range 1-42).

\section{DISCUSSION}

This study demonstrates that, in two separate follow-up programs for melanoma-prone families with CDKN2A mutations, melanomas among CDKN2A wt family members were seen at very comparable frequencies (Table 1). 
Table 2 Characteristics of phenocopies ${ }^{\mathrm{a}}$ and their children in Swedish and US melanoma families with CDKN2A mutations

\begin{tabular}{|c|c|c|c|}
\hline Phenocopies & $\begin{array}{l}\text { Phenocopies from } \\
\text { Swedish families } \\
n=9\end{array}$ & $\begin{array}{l}\text { Phenocopies from US } \\
\text { families } \\
n=10\end{array}$ & $\begin{array}{l}\text { Total } \\
n=19\end{array}$ \\
\hline Age (years) at first melanoma diagnosis, median (range) & $47(18-68)$ & $46(25-67)$ & $46(18-68)$ \\
\hline Female & $5(55.6 \%)$ & $4(40.0 \%)$ & $9(47.4 \%)$ \\
\hline Male & $4(44.4 \%)$ & $6(60.0 \%)$ & $10(52.6 \%)$ \\
\hline \multicolumn{4}{|l|}{ Retrospective and prospective melanomas, $n(\%)$} \\
\hline Melanoma after start of follow-up & $3(33.3 \%)$ & $3(30.0 \%)$ & $6(30 \%)$ \\
\hline \multicolumn{4}{|l|}{ Melanoma and cancer diagnoses, $n(\%)^{\mathbf{b}}$} \\
\hline Multiple primary melanoma & $1(11.1 \%)$ & $0(0.0 \%)$ & $1(5.3 \%)$ \\
\hline Squamous cell skin cancer & $0(0.0 \%)$ & $1(10 \%)$ & $1(5.3 \%)$ \\
\hline Non-skin cancer & $0(0.0 \%)$ & $2(20 \%)$ & $2(10.5 \%)$ \\
\hline Children of phenocopies & $n=28$ & $n=15^{c}$ & $n=43$ \\
\hline Age (years) at census date, median (range) & $20(3-57)$ & $35(5-51)$ & $25(3-57)$ \\
\hline \multicolumn{4}{|l|}{ Melanoma and cancer diagnoses, $n(\%)^{\mathbf{b}}$} \\
\hline Melanoma & $0(0.0 \%)$ & $0(0.0 \%)$ & $0(0.0 \%)$ \\
\hline Squamous cell skin cancer & $0(0.0 \%)$ & $1(6.7 \%)$ & $1(2.3 \%)$ \\
\hline Non-skin cancer & $0(0.0 \%)$ & $0(0.0 \%)$ & $0(0.0 \%)$ \\
\hline
\end{tabular}

${ }^{a}$ CDKN2A wild type melanoma cases from CDKN2A mutated melanoma families. ${ }^{\text {b}}$ The US phenocopy cases and their children each had one member diagnosed with basal cell carcinoma (BCC). BCCs were not accounted for in the Swedish families since BCCs are not registered in the Swedish Cancer Registry. ${ }^{\mathrm{C}}$ Among a total of 22 children, follow-up data were available for 15.

Interestingly, the phenocopy melanoma cases were diagnosed at young median ages (47 and 46 years), compared with melanoma cases in the Swedish and US general population (median age $>60$ years), ${ }^{12,13}$ but approximately 10 years later than among the melanoma cases with CDKN2A mutations from these two sets of families. ${ }^{1,2,14}$ The frequency of multiple primary melanomas in the phenocopies was also much lower (5.3\%) compared with what is seen among CDKN2A mutation carriers $(\sim 40 \%){ }^{14,15}$ This observation does not appear to have been biased by survival differences since all but one of the phenocopy melanoma cases were still alive at the end of follow-up.

Most melanomas among the phenocopies had been diagnosed prior to the identification of their respective family at each site. Nonetheless, in Sweden, the prospective risk for melanomas was also significantly higher among the CDKN2A wt subjects compared with population-based controls, although the risk increase was much lower than what is seen among $C D K N 2 A$ mutation carriers. ${ }^{2}$ These results suggest that $C D K N 2 A$ wt relatives of $C D K N 2 A$ mutation carriers have melanoma risk and age at diagnosis that is intermediate between population-based cases and CDKN2A mutation carriers. This observation supports the premise that other factors, in particular risk-modifying genes and ultraviolet exposure patterns, affect the melanoma penetrance of CDKN2A mutation carriers along with the melanoma risk among the CDKN2A wt family members. ${ }^{11,16,17}$ Consistent with this hypothesis, in a study of 815 CDKN2A mutation carriers from centers across Europe, North America, and Australia, variants in the $M C 1 R$ gene were significantly associated with melanoma risk in carriers across all three continents. ${ }^{17}$ CDKN2A mutation carriers with certain single $M C 1 R$ variants had significantly elevated odd ratios for melanoma (odds ratio up to 4.7) and two or more variants had odds ratios even higher. Hence, it is likely that many CDKN2A families that are identified for their melanoma propensity also have melanoma-associated variants in $M C 1 R$ and likely other low-risk melanoma susceptibility genes such as TYR, TYRP, and ASIP. In this study, we did not add data on any low-risk melanoma susceptibility gene variants, but it is likely that such variants play a role in the occurrence of melanoma among noncarriers of their family's CDKN2A mutation. Several additional high-risk melanoma susceptibility genes have been identified, including CDK4, POT1, $B A P 1, T E R F 2 I P$, and TERT promoter. ${ }^{18}$ Since mutations in $C D K N 2 A$ and these high-risk melanoma genes are extremely rare in the population it is very unlikely that such mutations would co-occur in the same family. Hence, although the families in this study have not been screened for these genes, it is unlikely that such high-risk mutations would be involved in the melanoma risk for phenocopies in the CDKN2A mutated families. 
Further, it is possible that the ascertainment of melanoma families and dermatologic follow-up results in increased rates of melanoma phenocopies; however, despite differing programs for inclusion and follow-up in Sweden and the United States, very similar phenocopy rates were seen at both sites. In contrast to what has been observed and reported among CDKN2A mutation carriers, ${ }^{2,3,19,20}$ the CDKN2A wt family members in this study did not appear to have elevated risks of nonmelanoma cancers. In addition, the offspring of the CDKN2A wt members did not show any evidence for increased risks of melanoma and other cancers. Of note, basal cell carcinomas were not included in the risk analyses since these tumors are not registered in the Swedish Cancer Registry. In this study, the presence of phenocopies was studied among 61 families with CDKN2A mutations, but similar studies involving other cohorts of CDKN2A mutated families are needed to confirm our findings.

When offering genetic testing to families it is important to also have knowledge of the outcomes of individuals who test negative for their family's mutation and to our knowledge this study is the first to address this problem in CDKN2A mutated families. The findings from this study may therefore be helpful for clinicians and genetic counselors who encounter families that carry CDKN2A mutations. To conclude, members of CDKN2A mutation carrying families who test negative for their family's $C D K N 2 A$ mutation remain at moderately increased risk for melanoma (but not for other cancers) and should therefore, in addition to being considered for continuing dermatologic surveillance, be encouraged to follow sun safety recommendations and practice skin selfexams.

\section{SUPPLEMENTARY MATERIAL}

Supplementary material is linked to the online version of the paper at http://www.nature.com/gim

\section{ACKNOWLEDGMENTS}

The work was supported in part by grants from the Swedish Cancer Society, the Radiumhemmet Research Funds, and Regional Funds and Hospital Funds in Lund and Stockholm. The work was supported in part by the Intramural Research Program of the Division of Cancer Epidemiology and Genetics, National Cancer Institute, National Institutes of Health. We are indebted to the participating families, whose generosity and cooperation made this study possible. We acknowledge the contributions to this work that were made by Diana Lindén, Lena Westerberg, Anita Schmidt-Zander, Rainer Tuominen, Johan Hansson, Virginia Pichler, Deborah Zametkin, Mary Fraser, and Barbara Rogers.

\section{DISCLOSURE}

The authors declare no conflict of interest.

\section{REFERENCES}

1. Goldstein AM, Struewing JP, Chidambaram A, Fraser MC, Tucker MA. Genotype-phenotype relationships in U.S. melanoma-prone families with CDKN2A and CDK4 mutations. J Natl Cancer Inst 2000;92:1006-10.

2. Helgadottir $\mathrm{H}$, Hoiom $\mathrm{V}$, Jonsson $\mathrm{G}$, et al. High risk of tobacco-related cancers in CDKN2A mutation-positive melanoma families. J Med Genet 2014;51:545-52.

3. de Snoo FA, Bishop DT, Bergman W, et al. Increased risk of cancer other than melanoma in CDKN2A founder mutation (p16-Leiden)-positive melanoma families. Clin Cancer Res 2008;14:7151-7.

4. Smith A, Moran A, Boyd MC, et al. Phenocopies in BRCA1 and BRCA2 families: evidence for modifier genes and implications for screening. $J$ Med Genet 2007;44:10-5.

5. Evans DG, Howell A. Breast cancer risk for noncarriers of family-specific BRCA1 and BRCA2 mutations: more trouble with phenocopies. J Clin Oncol 2012;30:1142-3; response, 3-4.

6. Kurian AW, Gong GD, John EM, et al. Breast cancer risk for noncarriers of family-specific BRCA1 and BRCA2 mutations: findings from the Breast Cancer Family Registry. J Clin Oncol 2011;29:4505-9.

7. Vos JR, de Bock GH, Teixeira N, et al. Proven non-carriers in BRCA families have an earlier age of onset of breast cancer. Eur J Cancer 2013;49: 2101-6.

8. Hansson J, Bergenmar M, Hofer PA, et al. Monitoring of kindreds with hereditary predisposition for cutaneous melanoma and dysplastic nevus syndrome: results of a Swedish preventive program. I Clin Oncol 2007;25: 2819-24.

9. The Swedish Cancer Registry. Socialstyrelsen. 2015. Accessed on 24 August 2017. http://www.socialstyrelsen.se/register/halsodataregister/ cancerregistret/inenglish.

10. Multi-Generation Register 2010. A description of contents and quality. Statistics Sweden (Statistiska Centralbyrån). 2011. Accessed on 24 August 2017. http://www.scb.se/statistik/_publikationer/BE9999_ 2011A01_BR_BE96BR1202.pdf.

11. Yang $X R$, Pfeiffer RM, Wheeler $W$, et al. Identification of modifier genes for cutaneous malignant melanoma in melanoma-prone families with and without CDKN2A mutations. Int J Cancer 2009;125: 2912-7.

12. Ingvar C, Eriksson H. Melanoma incidence in Sweden. Lakartidningen 2017;114: 884-886.

13. Howlader N, Noone AM, Krapcho M, et al. (eds). SEER Cancer Statistics Review, 1975-2014. National Cancer Institute: Bethesda, MD, 2017. Accessed on 24 August 2017. https://seer.cancer.gov/csr/1975_2014/.

14. van der Rhee Jl, Krijnen P, Gruis NA, et al. Clinical and histologic characteristics of malignant melanoma in families with a germline mutation in CDKN2A. J Am Acad Dermatol 2011;65:281-8.

15. Helgadottir $\mathrm{H}$, Hoiom $V$, Tuominen $\mathrm{R}$, et al. Germline CDKN2A mutation status and survival in familial melanoma cases. I Natl Cancer Inst 2016;108:djw135.

16. Bishop DT, Demenais F, Goldstein AM, et al. Geographical variation in the penetrance of CDKN2A mutations for melanoma. I Natl Cancer Inst 2002;94:894-903

17. Demenais F, Mohamdi H, Chaudru V, et al. Association of MC1R variants and host phenotypes with melanoma risk in CDKN2A mutation carriers: a GenoMEL study. J Natl Cancer Inst 2010;102:1568-83.

18. Read J, Wadt KA, Hayward NK. Melanoma genetics. J Med Genet 2015;53:1-14.

19. Goldstein AM, Struewing JP, Fraser MC, Smith MW, Tucker MA. Prospective risk of cancer in CDKN2A germline mutation carriers. I Med Genet 2004;41:421-4.

20. Goldstein AM, Chan $M$, Harland $M$, et al. High-risk melanoma susceptibility genes and pancreatic cancer, neural system tumors, and uveal melanoma across GenoMEL. Cancer Res 2006;66:9818-28. 